\title{
Design and Experimental Validation of an Integrated Multi-Channel Charge Amplifier for Solid-State Detectors with Innovative Spectroscopic Range Booster
}

\author{
Stefano Capra, Alberto Pullia
}

\begin{abstract}
The circuit structure and the experimental performance of a new integrated multi-channel charge-sensitive preamplifier for silicon or germanium detectors are shown. The circuit has been conceived and optimized for the silicon detector GAL-TRACE, employed for light and heavy ion spectroscopy in nuclear physics experiments. The chip includes four channels optimized for anodic signals and one channel specifically designed for cathodic signals. An $\mathrm{I}^{2} \mathrm{C}$ interface is used to adjust as needed a host of key parameters of the pre-amplifier, such as sensitivity and bandwidth. An integrated range booster yields an exceptional dynamic range of $103 \mathrm{~dB}$ for the cathodic channel. The circuit features a low-power consumption of $11 \mathrm{~mW}$ per channel, a fast rise-time of the order of $10 \mathrm{~ns}$, and low noise. Thanks to an accurate design of the input stage, the Equivalent Noise Charge, measured with a commercial shaping amplifier, is as low as $\mathbf{1 3 0}$ r.m.s. electrons at a shaping time of $6 \mu \mathrm{s}$ with $5 \mathrm{pF}$ of detector capacitance. The bandwidth of the preampifier is adequate for pulse-shape-analysis techniques used for particle discrimination.
\end{abstract}

Index Terms-Charge-Sensitive amplifier, Analog integrated circuits, Spectroscopy, Particle Detectors, Pulse Analysis, LowNoise electronics.

\section{INTRODUCTION}

G AL-TRACE [1] is a silicon detector array of ionizing particles to be used as ancillary for large gamma spectrometers like AGATA [2] and GALILEO [3] in nuclear physics experiments with radioactive ion beams [4], [5]. Its main purposes are reaction-channel selection and Doppler correction on gamma spectra. These are achieved performing particle identification and high-resolution ion spectroscopy. This array is part of the wider European research project GRIT [6].

The standard technique adopted to discriminate between different particles requires the use of two detectors in a telescopic configuration. The impinging particles pass through the first, thin one $(\Delta \mathrm{E})$ and are stopped in the second, thick one (E). The ratio between the amount of energy released in the first and the second detector, together with the information of the total particle energy, allows to derive the physical properties of the charged particle thanks to the Bethe-Bloch formula. This approach is effective but is limited by the minimum energy

S. Capra is with the Department of Physics, University of Milano and INFN of Milano, Italy (email: stefano.capra@unimi.it).

A. Pullia is with the Department of Physics, University of Milano, Italy, and INFN of Milano, Italy (email: alberto.pullia@mi.infn.it). required by the particle to run through the first detector layer without being fully stopped.

In order to reduce the energy threshold for particle discrimination down to 5-10 keV, the GAL-TRACE array will implement Pulse-Shape Analisys (PSA) techniques [7]. These rely on the fact that particles with different mass and charge produce different ionization traces inside the silicon crystal. From an electronic point of view, it is possible to discriminate protons, deuterons, tritons, alphas and other light ions analyzing the leading edge's shape of the pre-amplifier signals. More specifically, the analysis requires to create a figure of merit that depends on the duration and the amplitudes of the electron (fast) and hole (slow) components of the detector's current signals. This can be performed only if the pre-amplifier's bandwidth is wide enough to preserve the highfrequency information coming from the detector. In the case of GAL-TRACE, it has been estimated that, using a $200 \mu \mathrm{m}$ thick detector, a pre-amplifier's rise-time of $10 \mathrm{~ns}$ or lower is required. A linear dynamic range of $40 \mathrm{MeV}$ is advisable, corresponding to an input charge of $1.8 \mathrm{pC}$ : gain should be properly selected to adapt the energy range requirement on the available output voltage swing. The latter should be at least $2 \mathrm{~V}$. Above this threshold PSA is not required, but the possibility to extend the spectroscopic range of another order of magnitude can be useful in some contexts.

The GAL-TRACE silicon detectors are segmented in 60 $4 \times 4 \mathrm{~mm}^{2}$ square pads, arranged in a $12 \times 5$ configuration. The front anodic electrodes have an estimated capacitance of $4 \mathrm{pF}$, while the capacitance of the cathodic (back) electrode is of about $50 \mathrm{pF}$. The pre-amplifiers must be optimized for such values.

In the foreseen experimental setup the channel count is quite high. In the pursue of the highest solid-angle coverage, not less than four telescopes should be used: hundreds of readout channels must be packed inside the relatively small volume of the scattering chamber. The front-end electronics must be very compact and suited to work in vacuum. For these reasons, the adoption of integrated solutions with small form-factor and low power dissipation is recommended: less than $15 \mathrm{~mW} /$ channel.

We designed, realized and tested an integrated multichannel charge-sensitive pre-amplifier [8], [9] compliant with the specification of the GAL-TRACE detector. The circuit peculiar features are low noise, low power consumption, fast 


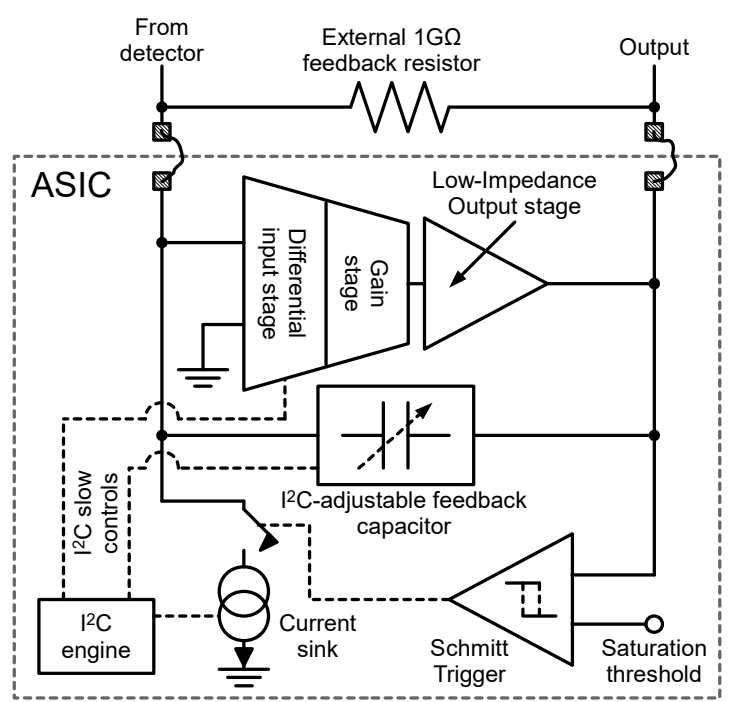

Fig. 1. Architecture of one channel of the multi-channel CSP ASIC for GAL-TRACE. The feedback resistor is the only discrete component, while all the others are integrated inside the chip. The operational amplifier has been divided in stages for better clarity. The input differential stage ensures a $0 \mathrm{~V}$ output quiescent voltage and, together with the gain stage, provide a $10^{5}$ open-loop gain. The output stages is able to drive a $50 \Omega$-terminated coaxial line. The comparator and the current sink together constitute the fast-reset device that enables the spectroscopic range boost.

rise-time, and wide dynamic range. An innovative auxiliary device extends the natural spectroscopic range of the preamplifier by more than one order of magnitude, allowing for high-resolution spectroscopy above the natural pre-amplifier saturation limit. An embedded $\mathrm{I}^{2} \mathrm{C}$ engine allows to adjust a host of key parameters via digital slow control. In this way the pre-amplifier can work properly with very different detector capacitances and in different experimental conditions, ensuring best rise-time and low ringing in each case.

The chip, realized in AMS C35 technology [10] includes four front channels optimized for anodic signals and one back channel optimized for cathodic signals and occupies an area of $5 \mathrm{~mm}^{2}$.

\section{CIRCUIT STRUCTURE}

The core of this circuit is an operational amplifier with a low-noise input stage, a DC gain in the order of $10^{5}$ and an output stage able to drive a $50 \Omega$-terminated coaxial cable (see Fig. 1). The feedback capacitor is integrated in the chip but the feedback resistor is left as an external component. An integrated circuit structure acting as a large-value resistor [11] is being developed, but for this prototype a more conservative approach was chosen. The external feedback device installed is a $1 \mathrm{G} \Omega$ thin-film chip resistor as little as $1 \times 0.5 \mathrm{~mm}^{2}$ (0402 case) which guarantees noise and linearity specifications suitable for the foreseen application.

The feedback capacitor is adjustable in a choice of four different values. The value of this component is a key parameter because, besides determining the pre-amplifier sensitivity, it greatly impacts on circuit stability and speed.

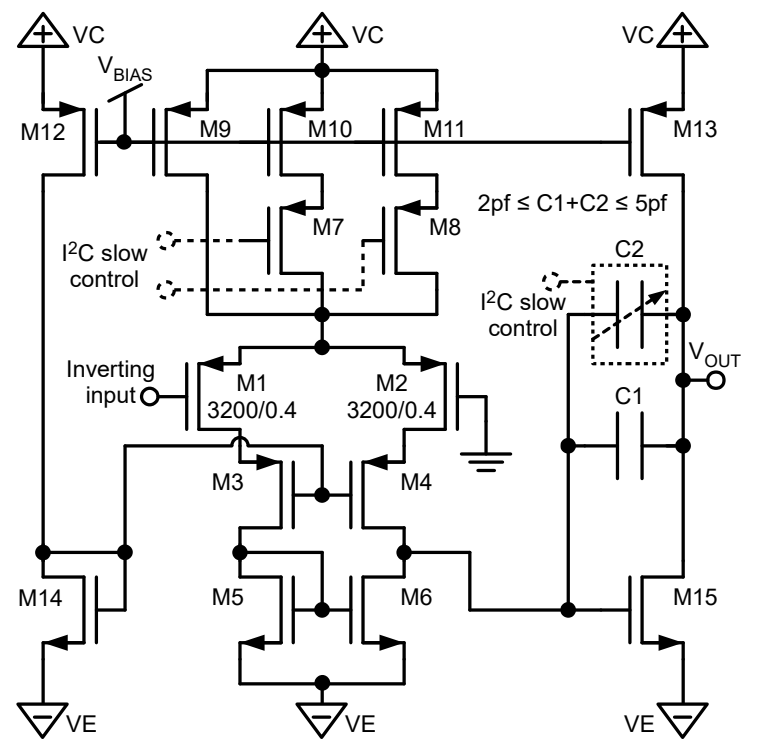

Fig. 2. Simplified schematic of the input and gain stage with adjustable bias current and Miller capacitor. The switches represent transmission gates directly activated from the logic lines of the $\mathrm{I}^{2} \mathrm{C}$ engine. M1-4 are $3.3 \mathrm{~V}$-tolerant while the others are $5 \mathrm{~V}$-tolerant.

A dedicated circuit structure swiftly resets the pre-amplifier when the output voltage approaches saturation [12], [13]. It consists of a Schmitt Trigger Comparator (STC) and a controlled Current Sink (CS). The STC monitors the output voltage level and activates the CS when it crosses a preset threshold. The precise constant current CS draws charge from the input node, yielding a fast reset of the pre-amplifier. This circuit solution is mainly used to reduce the system dead time, but can be also exploited in an original way as a spectroscopic range booster as described in literature [14]. This technique is described in the next section.

The pre-amplifier is powered with a dual $\pm 2.5 \mathrm{~V}$ supply. Thanks to an accurate design, a power consumption has been obtained as low as $11 \mathrm{~mW} / \mathrm{ch}$, which meets the GAL-TRACE requirements.

\section{A. INPUT AND GAIN STAGE}

The input stage of the CSP (see Fig. 2) is differential, with the non-inverting input hard-wired to ground, which yields a precise quiescent output voltage of $0 \mathrm{~V}$. It is well known that the many benefits of a differential structure are counterbalanced by some drawbacks, including a larger noise and a lower speed. Eventually, we opted for a differential structure mainly because the output stage has to drive a $50 \Omega$ terminated coaxial cable and a $0 \mathrm{~V}$ quiescent level at the preamplifier output is necessary to minimize the static power dissipation. A single-ended input structure with a $0 \mathrm{~V}$ preamplifier's output DC point would have required an active transconductor as feedback device or a physical resistance driven by some voltage shifter. The first solution was discarded because such devices are noisier than a physical $1 \mathrm{G} \Omega$ resistors [15]. The second solution was discarded in order to avoid any possible signal deformation than can corrupt the spectroscopic 
performance of the system when applying Moving-Window Deconvolution (MWD) algorithms [16].

The bias point and the physical dimensions of the input transistors M1 and M2 have been carefully chosen so as to optimize speed and noise performance, while also trying to keep their $1 / \mathrm{f}$ noise low. In nuclear spectroscopy this noise component gives a contribution to the total equivalent noise charge of the pre-amplifier that doesn't depend on the chosen shaping time.

The ASIC includes four channels optimized for anodic signals and one, separately powered, for cathodic signals. The latter, meant to acquire the signals from the back electrode of the detector, is similar to those of the front channels but is based on a complementary design. The only remarkable difference is the size of the input transistors, that was chosen to be $3200 / 0.8 \mu \mathrm{m}$ after a noise optimization procedure.

The low-frequency gain $G_{L F}$ can be calculated as the product of the input-stage gain $G_{I S}$ and the second-stage gain $G_{S S}$. The transconductance and the output resistance of the transistor $\mathrm{X}$ are respectively written $g_{m x}$ and $R_{O x}$.

$$
\begin{aligned}
G_{L F} & =G_{I S} \cdot G_{S S} \\
& =\left[g_{m 1,2}\left(R_{O 6} \| g_{m 4} R_{O 4} R_{O 2}\right)\right] \cdot\left[g_{m 15}\left(R_{O 15} \| R_{O 13}\right)\right]
\end{aligned}
$$

Considering $Z_{1}=R_{O 6} \| g_{m 4} R_{O 4} R_{O 2}$ and $Z_{2}=R_{O 15} \|$ $R_{O 13}$, the exact transfer function between opamp input and output is:

$$
\frac{v_{O U T}(s)}{v_{I N}(s)}=-g_{m 1,2} Z_{1} g_{m 15} Z_{2} \cdot \frac{1-s C \frac{1}{g_{m 15}}}{1+s C\left(K \cdot Z_{1}+Z_{2}\right)}
$$

where $K=1+g_{m 15} Z_{2}$ is the Miller amplification factor of the second stage.

\section{B. OUTPUT STAGE}

The output stage (see Fig. 3) nested in the loop structure is an evolution of the classic White follower [17]. In order to understand the working principle, let's first ignore the role of the bias network and let's consider only $\mathrm{I}_{2}, \mathrm{I}_{4}$ and the transistors M1, M3, M4. The transistor M3 carries the difference between $\mathrm{I}_{2}$ and the current of M1. This current difference is injected on the gate of M4 and "compared" with $\mathrm{I}_{4}$. A change in $\mathrm{V}_{I N}$ would naturally induce a variation of the M1 current, but, thanks to the local feedback loop, the driver transistor M4 ensures a constant current through M1. Thanks to this solution, the current of M1 is constant even when driving a $100 \Omega$ load to negative voltages.

A simple circuit as the one just described would work fine only for negative output voltages. When the output signal goes few tens of $\mathrm{mV}$ over $0 \mathrm{~V}$, the circuit requires $\mathrm{M} 1$ to drive the $100 \Omega$ load with a current that is higher than the one of $\mathrm{I}_{2}$. This fact not only would shut down M3 (that in turn would shut down M4), but also would greatly limit the positive output voltage swing to $\mathrm{I}_{2} \cdot 100 \Omega$. To enhance the positive dynamic range, M2 is inserted in parallel with $\mathrm{M} 1$, since its current is not limited to $\mathrm{I}_{2}$.

This solution ensures some hundreds of $\mathrm{mV}$ of positive dynamic range (that is more than enough for the foreseen

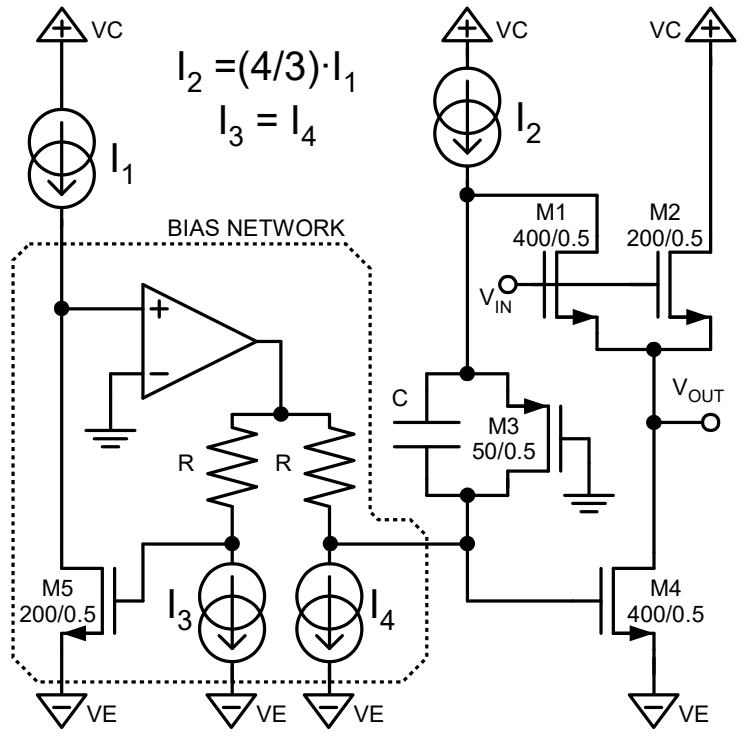

Fig. 3. Simplified schematic of the output stage. The feedback network provided by M3, C and the bias network ensure that M4 drains a well defined minimum current. This prevents M4 from being shut off if the output voltage runs over $0 \mathrm{~V}$ when driving a $100 \Omega$ load.

application), but still doesn't solve another problem. If a signal from the detector requires the output voltage to abruptly fall from a positive baseline to a new voltage level under $0 \mathrm{~V}$, this would require a prompt reaction of M3 and M4, but these transistors with a positive baseline would be completely turned off. The time required for them to turn on again is comparable to or higher than the typical rise-time of the detector signals. Simulations and experimental tests on previous pre-amplifier prototypes demonstrate that the $\mathrm{M} 3-\mathrm{I}_{4}-\mathrm{M} 4$ feedback loop takes several tens of nanoseconds to turn on and the leading edge of signals crossing the $0 \mathrm{~V}$ level is characterized by longer rise-time, distorted shape and appreciable ringing ${ }^{1}$.

To solve this issue the bias-network circuit was added. This one has the specific role of ensuring that M4 is never completely turned off when the output voltage is equal to $0 \mathrm{~V}$ or higher. In fact, when no current flows through $\mathrm{M} 3$ the source-to-gate voltage of M4 is equal to the one of M5 and these two transistors are in a precise geometrical relationship, leading to a minimum predictable current on M4 (2. $\mathrm{I}_{1}$ in our specific case). The local feedback loop responds promptly to input-voltage variations even if M3 is in "off" state because this transistor is bypassed by $\mathrm{C}$ during the M3 startup time.

The choice of a low-impedance output stage makes the loop gain and the pre-amplifier dynamic properties largely insensitive to the output load.

Due to the negative charge-to-voltage conversion factor $\left(V_{o} / Q_{i n}\right)$ of the channels, the output voltage swing is $-2.4 \mathrm{~V}$ to $+0.5 \mathrm{~V}$ for the front channels (anodic signal) and from $-0.5 \mathrm{~V}$ to $+2.4 \mathrm{~V}$ for the back channel (cathodic signal), obtained with a complementary output stage design.

\footnotetext{
${ }^{1}$ Please notice that this is not an issue if this output stage is implemented in a hybrid pre-amplifier with a JFET as input transistor [18], in which case the DC quiescent baseline lies by default some hundreds of $\mathrm{mV}$ under the $0 \mathrm{~V}$ threshold.
} 


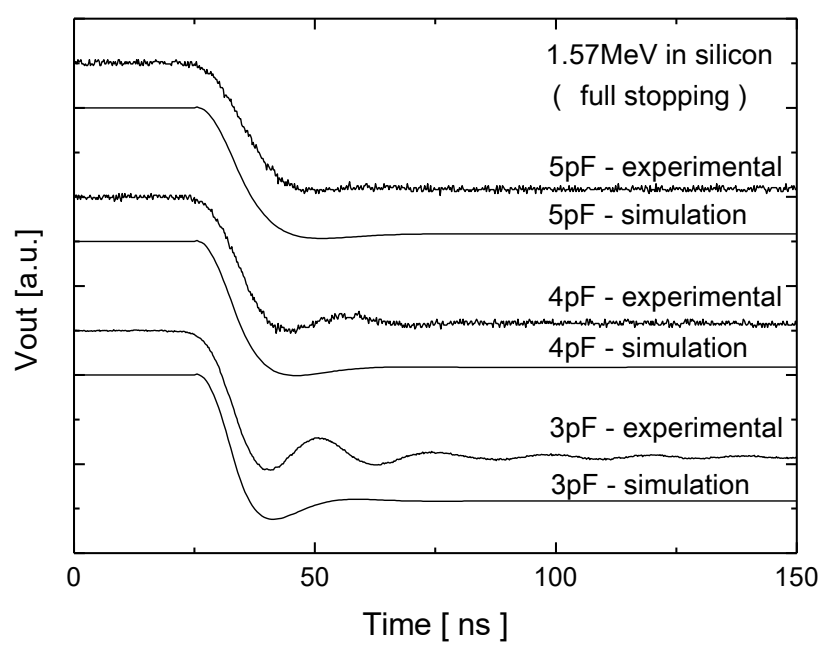

Fig. 4. CSP output waveforms obtained with the same test pulse equivalent to a full-stopping $1.57 \mathrm{MeV}$ event in a silicon detector $\left(436 \cdot 10^{3}\right.$ electrons released). The CSP was set with three different bandwidth configurations. The configuration with $3 \mathrm{pF}$ of Miller compensation capacitance is too fast for a $4 \mathrm{pF}$ detector capacitance and shows ringing. The configuration with $4 \mathrm{pF}$ of Miller compensation capacitance instead is a good trade off between speed and ringing. The signals were shifted along the Y-axis for better visualization.

\section{RISE-TIME OPTIMIZATION}

A useful feature of the ASIC CSP is the possibility to adjust some critical parameters via slow control through a simple digital $\mathrm{I}^{2} \mathrm{C}$ interface, such as the Miller compensation capacitance. The preamplifier bandwidth can thus be optimized for different detector types: it may be used in conjunction with Silicon Pad and Strip Detectors or even with Germanium Crystals [19], [20].

The PSA performed with GAL-TRACE detectors requires a CSP rise-time of no more than $10 \mathrm{~ns}(10 \%-90 \%)$ [7]. With such fast rise-time it is very difficult to avoid unwanted signal ringing, which, in turn, can corrupt the information contained in the detector signals and thus in the pre-amplifier signals' leading edge. At the same time, a slow pre-amplifier masks completely the high-frequency information coming from the detector. The best experimental condition for PSA is a tradeoff between speed and ringing. In this regard, the possibility to adjust the bandwidth via slow control is paramount. Just to give an example, in Fig. 4 three output waveforms are depicted. They were obtained connecting the programmable CSP to a pulser and varying the bandwidth of the pre-amplifier. The tests where performed mounting the ASIC on a custom designed PCB. The detector was simulated with a squarewave pulser, a series test capacitor of $1 \mathrm{pF}$ and a $\sim 4 \mathrm{pF}$ parallel capacitor connected between the input node of the pre-amplifier and ground, which stands for the capacitance of the junction.

Other programmable parameters are the value of the feedback capacitance, the Fast-Reset speed and the bias current of the input stage.

\section{SPECTROSCOPIC RANGE BOOSTER}

The spectroscopic energy range of a pre-amplifier is naturally limited by its output voltage swing. It is worth to notice

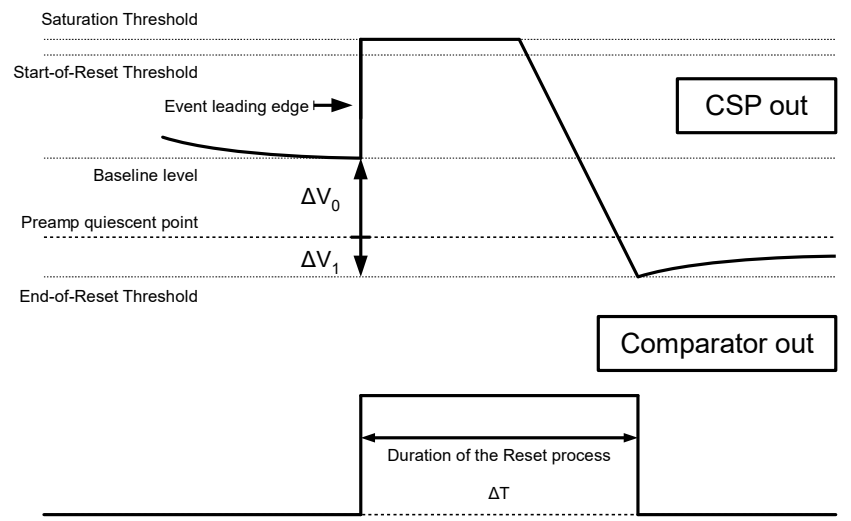

Fig. 5. Conceptual drawing of a reset procedure. The upper signal is the pre-amplifier output while the lower one is the comparator square wave.

that when the pre-amplifier saturates, the charge released by the detector is not lost: it remains trapped on the stray capacitances of the input node. If it doesn't find parasitic resistive paths to ground it can still be collected and measured. This is the core idea of the proposed spectroscopic range booster: in case of saturation we discharge the input node with a constant and controlled current generator and, since there is a linear relationship between charge and duration of the reset process, we are able to retrieve the energy information even if the preamplifier output signal is heavily distorted.

The reset phase is activated by a Schmitt trigger: it starts when the preamplifier output signal runs above a pre-defined threshold (namely the saturation threshold) and ends when the default preamplifier output DC voltage is restored. The linearity and resolution of this spectroscopic technique relies heavily on the current generator used to collect the charge from the input node. For this reason, the resistor that generates such current is biased with a constant voltage controlled by a dedicated operational amplifier. The switches that connect the current generator to the input node are designed to avoid charge leak and ensure negligible charge injections during the switching transients.

In order to visualize graphically the quantities under discussion, please refer to Fig. 5. The comparator that activates the current sink also produces an auxiliary logic signal that remains active from the beginning to the end of the reset process. Being $I_{R E S}$ the reset current and $C_{F}$ the feedback capacitor, the time width $\Delta T$ of the square pulse produced by the comparator is equal to:

$$
\Delta T=\left(\frac{Q+Q_{0}-Q_{1}}{I_{R E S}}\right)
$$

where $Q$ is the charge released by the detector, $Q_{0}$ is the residual charge on the input node due to previous events (equal to $C_{F} \cdot \Delta V_{0}$ ) and $Q_{1}$ is a fixed charge contribution due to any possible misalignment between the pre-amplifier quiescent point and the "End-of-Reset" threshold (equal to $\left.C_{F} \cdot \Delta V_{1}\right)$. Given the nature of the pre-amplifier input stage, the "End-of-Reset" threshold is set to $0 \mathrm{~V}$. The entity of $Q_{1}$ is thus determined by the pre-amplifier input-voltage offset. 
While $Q_{1}$ is a constant term that can be canceled out through proper energy calibration, $Q_{0}$ constitutes a variable term depending on the baseline preceding the event. The fast-reset procedure is active only for high-energy events: those above the saturation threshold of the pre-amplifier. In low-countingrate situations the term $Q_{0}$ is thus completely negligible and the energy-retrieval technique is perfectly functional. In case of high counting rates such term may become relevant and more refined algorithms [9] are required for high-resolution spectroscopy. A circuit implementing an analog algorithm that corrects the fast-reset spectra has also been realized [21]. It proved to reduce dependency of $\Delta T$ on $Q_{0}$ to a secondorder effect and enables the fast-reset circuit to be used also in high-counting-rate conditions. All the fast-reset energy measurements in this work have been performed evaluating the reset duration only.

This approach is the evolution of the one described in [12], but with a relevant difference. The cited work is about a twostage discrete-type circuit, where the reset technique is used to limit the dynamic range of the second stage adapting it to the input range of an ADC: in order to be effective, the first stage that performs the charge integration should never saturate. On the other hand, the proposed pre-amplifier is an integrated one with the fast-reset directly applied to the chargeintegration stage. In this case the reset technology is exploited to overcome the circuit saturation limit.

\section{MEASUREMENT OF THE EQUIVALENT NOISE CHARGE AND COMPARISON WITH NOISE SIMULATIONS}

The experimental equivalent noise charge (ENC) was evaluated with the following setup. The pulser was connected to the pre-amplifier through a test capacitor of $1 \mathrm{pF}$. A $4 \mathrm{pF}$ test capacitor was connected between the input node and ground to simulate the junction capacitance of the detector. The output of the CSP was connected to an ORTEC 572 shaping amplifier. The quasi-Gaussian signals thus obtained were recorded with a digital scope. The pulser was programmed to simulate a $1 \mathrm{MeV}$ event inside the detector, that corresponds to a charge pulse of $278 \cdot 10^{3}$ electrons. The pulse produced by the shaping amplifier was then adjusted to a preset amplitude $(6 \mathrm{~V})$ by proper setting of the amplifier gain. The pulser was then disconnected. The noise produced by the CSP and filtered by the shaping amplifier was recorded with the digital scope. The ENC was calculated from the r.m.s. values of the random voltage fluctuations and then listed in a table against the corresponding shaping times. This procedure was repeated for every channel of the pre-amplifier and for 6 different shaping times between $0.5 \mu \mathrm{s}$ and $10 \mu \mathrm{s}$.

With the simulator it is possible to calculate the inputreferred equivalent power density of noise $\left(i_{\text {noise }}^{2}(\omega)\right)$ of the circuit taking into account the extracted post-layout parasitics (see Fig. 6). We can use these data to estimate the ENC. Being $S(\tau, \omega)$ the normalized transfer function of an ideal 5-pole shaping amplifier with shaping time $\tau$, the ENC can be easily

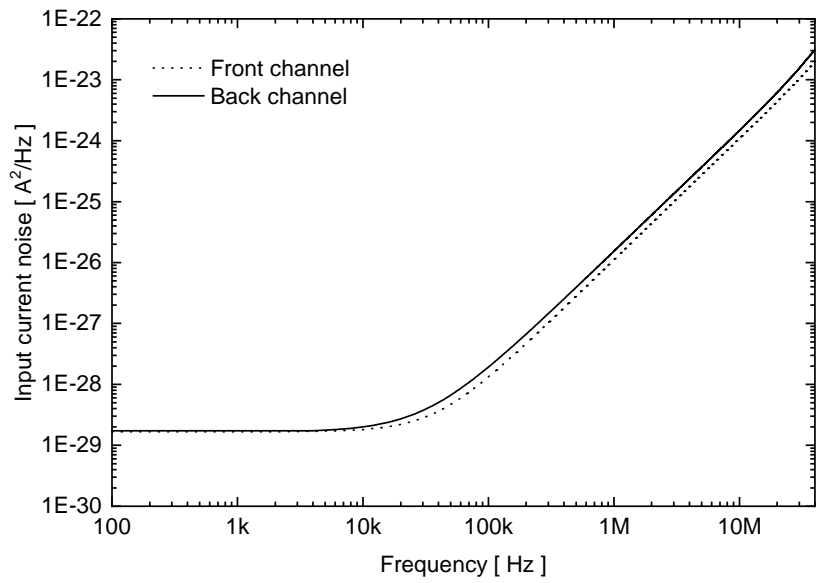

Fig. 6. Input current noise with $5 \mathrm{pF}$ of detector capacitance. Computer simulations.

calculated with the expression in 4.

$$
E N C=\sqrt{\int_{0}^{+\infty} i_{\text {noise }}^{2}(\omega) \cdot|S(\tau, \omega)|^{2} d \omega} .
$$

The results, reported in Tab. I, show a natural spread of the ENC values from channel to channel, but always in reasonable agreement both with GAL-TRACE requirements

TABLE I

EQUIVALENT NOISE CHARGE OF THE FRONT AND BACK CHANNELS IN ELECTRONS RMS AND EQUIVALENT ENERGY IN SILICON (FWHM). THE OPTIMAL SHAPING TIME IS BETWEEN 6 AND $10 \mu$ S FOR ALL CHANNELS. THE TOTAL INPUT-REFERRED CAPACITANCE IS $\sim 5 \mathrm{PF}$ AND THE FEEDBACK CAPACITANCE IS $1 \mathrm{PF}$.

\begin{tabular}{|c|c|c|c|c|}
\hline \multirow{2}{*}{$\begin{array}{c}\text { Shaping } \\
\text { Time } \\
{[\mu \mathrm{s}]}\end{array}$} & \multicolumn{2}{|c|}{ Channel 1} & \multicolumn{2}{|c|}{ Channel 2} \\
\hline & $\begin{array}{l}\mathrm{e}^{-} \\
\mathrm{rms}\end{array}$ & $\begin{array}{c}\mathrm{keV}(\mathrm{Si}) \\
\text { FWHM }\end{array}$ & $\begin{array}{l}\mathrm{e}^{-} \\
\mathrm{rms}\end{array}$ & $\begin{array}{c}\mathrm{keV}(\mathrm{Si}) \\
\text { FWHM }\end{array}$ \\
\hline 0.5 & 189 & 1.60 & 244 & 2.07 \\
\hline 1 & 154 & 1.31 & 194 & 1.64 \\
\hline 2 & 132 & 1.12 & 160 & 1.36 \\
\hline 3 & 125 & 1.06 & 148 & 1.25 \\
\hline 6 & 124 & 1.05 & 139 & 1.18 \\
\hline 10 & 128 & 1.09 & 141 & 1.20 \\
\hline
\end{tabular}

\begin{tabular}{|c|c|c|c|c|c|c|}
\hline \multirow{2}{*}{$\begin{array}{c}\text { Shaping } \\
\text { Time } \\
{[\mu \mathrm{s}]}\end{array}$} & \multicolumn{2}{|c|}{ Channel 3} & \multicolumn{2}{|c|}{ Channel 4} & \multicolumn{2}{|c|}{ Simulation (front) } \\
\hline & $\begin{array}{l}\mathrm{e}^{-} \\
\mathrm{rms}\end{array}$ & $\begin{array}{c}\mathrm{keV}(\mathrm{Si}) \\
\text { FWHM }\end{array}$ & $\begin{array}{l}\mathrm{e}^{-} \\
\mathrm{rms}\end{array}$ & $\begin{array}{c}\mathrm{keV}(\mathrm{Si}) \\
\mathrm{FWHM} \\
\end{array}$ & $\begin{array}{l}\mathrm{e}^{-} \\
\mathrm{rms}\end{array}$ & $\begin{array}{c}\mathrm{keV}(\mathrm{Si}) \\
\text { FWHM }\end{array}$ \\
\hline 0.5 & 235 & 1.99 & 257 & 2.18 & 250 & 2.12 \\
\hline 1 & 185 & 1.57 & 203 & 1.72 & 187 & 1.59 \\
\hline 2 & 150 & 1.27 & 167 & 1.42 & 144 & 1.22 \\
\hline 3 & 138 & 1.17 & 154 & 1.31 & 128 & 1.09 \\
\hline 6 & 128 & 1.09 & 144 & 1.22 & 120 & 1.02 \\
\hline 10 & 129 & 1.09 & 144 & 1.22 & 132 & 1.12 \\
\hline
\end{tabular}

\begin{tabular}{|c|c|c|c|c|}
\hline \multirow{2}{*}{$\begin{array}{c}\text { Shaping } \\
\text { Time } \\
{[\mu \mathrm{s}]}\end{array}$} & \multicolumn{2}{|c|}{ Back Channel } & \multicolumn{2}{|c|}{ Simulation (back) } \\
\hline & $\begin{array}{l}\mathrm{e}^{-} \\
\mathrm{rms}\end{array}$ & $\begin{array}{c}\mathrm{keV} \\
\text { FWHM }\end{array}$ & $\begin{array}{l}\mathrm{e}^{-} \\
\mathrm{rms}\end{array}$ & $\begin{array}{c}\mathrm{keV} \\
\text { FWHM }\end{array}$ \\
\hline 0.5 & 229 & 1.94 & 296 & 2.51 \\
\hline 1 & 188 & 1.59 & 224 & 1.90 \\
\hline 2 & 157 & 1.33 & 174 & 1.48 \\
\hline 3 & 147 & 1.25 & 154 & 1.31 \\
\hline 6 & 138 & 1.17 & 140 & 1.19 \\
\hline 10 & 136 & 1.15 & 148 & 1.25 \\
\hline
\end{tabular}




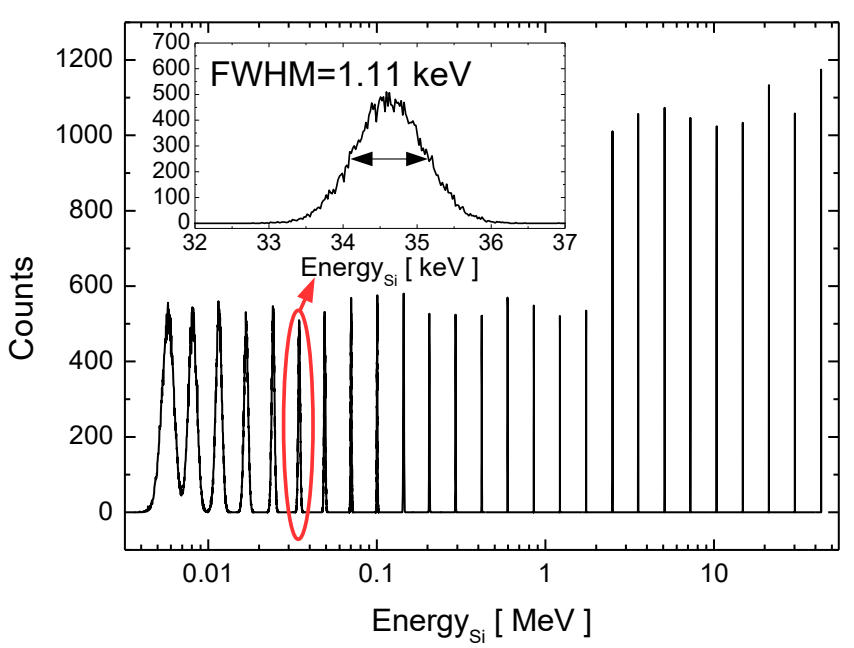

Fig. 7. A wide distribution of pulser lines obtained by sequentially changing the pulser signal height and histogramming the signal of the front channel. Note that the energy axis is in logarithmic scale. In the box in the upperleft corner the sixth peak is shown. The full width at half maximum of the peak at $34.6 \mathrm{keV}$ is equal to $1.11 \mathrm{keV}$, which is really good respect to the experimental requirements of the GAL-TRACE detector. The total inputreferred capacitance is $\sim 5 \mathrm{pF}$. This spectrum is a good representative of the average spectroscopic performance of the chip in the production run. For the "best-case-ever" please refer to Fig. 8.

and simulations. A reduction of the feedback capacitance from the value used in these tests $(1 \mathrm{pF})$ to lower values $(0.2 \mathrm{pF})$ can reduce slightly the ENC, although the effect of the detector's capacitance is predominant.

The reduction of the 1/f series noise was one of the main design goals. Simulations demonstrated that not only the input transistors but also other active devices of the input stage may contribute significantly to the overall $1 / \mathrm{f}$ series noise component of the ENC. Careful dimensioning and biasing of all the transistors was necessary to keep such noise under the desired level. The 1/f contribute to the total input-referred noise current at the noise corner frequency is lower than $2 \%$ in the front channel and lower than $13 \%$ in the back one.

\section{ACQUISITION OF PULSER-PRODUCED SPECTRA}

Beside analyzing the noise performances of the ASIC CSP with the aforementioned method, the pre-amplifier's performances have been evaluated with the acquisition of some spectra (see Fig. 7, 8 and 10). Please notice that, throughout this section, the equivalence between the energy deposited in a silicon detector and the corresponding released charge is done considering a ratio of 278 electrons $/ \mathrm{keV}$.

The experimental setup was the following. The pulser was programmed to simulate detector events equally spaced on an exponential scale between $7 \mathrm{keV}$ and $40 \mathrm{MeV}$. The output signal was filtered with an ORTEC 572 shaping amplifier with a shaping time of $6 \mu \mathrm{s}$ for the anodic channels and $10 \mu \mathrm{s}$ for the cathodic one. The amplitude of the shaped pulses was then digitized with an Ortec MCB 926 ADC. The resulting spectrum was saved on a PC with suitable software. This

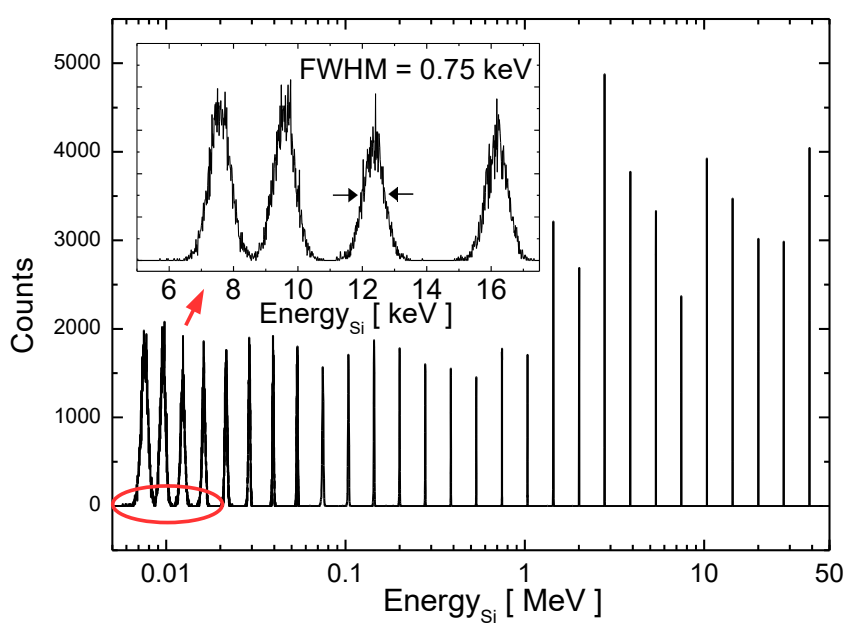

Fig. 8. An energy spectrum equivalent to the one in Fig. 7 that can be considered the "best-case-ever". The fluctuations of the technology parameters due to the fabrication process lead to different resolutions between different chips of the same production run.

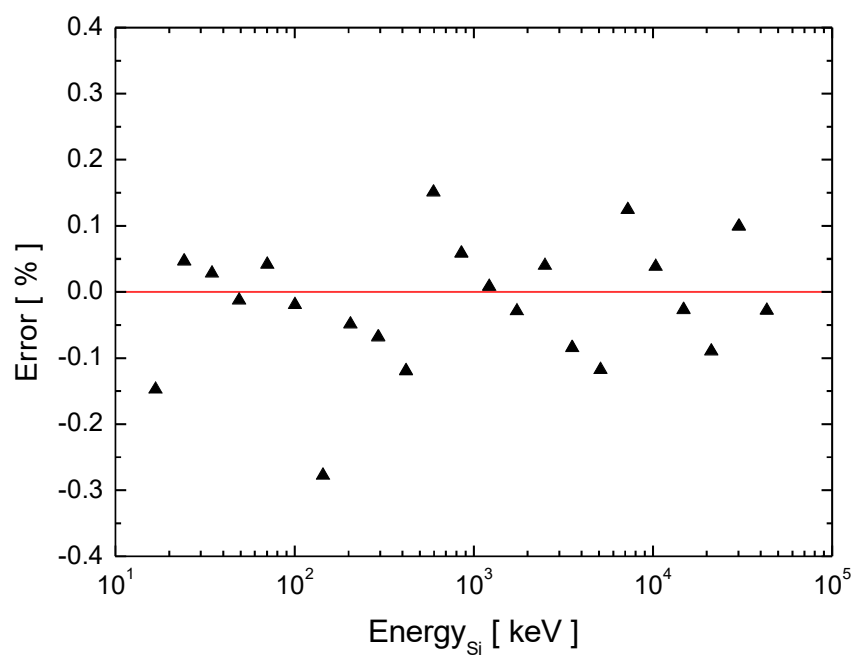

Fig. 9. Relative static error of the peaks in fig. 8. Their absolute value is always lower than $0.15 \%$ except one point. The four low-energy peaks have been omitted because for small pulses the non-linearity of the pulser becomes predominant.

procedure was adopted for signals under the pre-amplifier saturation threshold. Fig. 7 and 8 show the spectral lines obtained with this technique. Note that a logarithmic scale is used for the $\mathrm{x}$-axis, which helps appreciate details over a four-orders-ofmagnitude range. For this reason the width of the pulser lines seems to change depending on the line position. The actual width of the pulser lines seen over a linear scale, however, is uniform as expected. The experimental results show that the energy range of the ASIC extends up to $\approx 40 \mathrm{MeV}$ in silicon when using a $1 \mathrm{pF}$ feedback capacitance. The linearity of the pre-amplifier has been evaluated by plotting the centroids of the spectral lines in Fig. 8 vs the input-test-pulse amplitudes and fitting such data with a linear regression (see Fig. 9). The experimental results demonstrate that the circuit non-linearity across its dynamic range is within $\pm 0.1 \%$, which is compliant with the requirements of the GAL-TRACE array. 


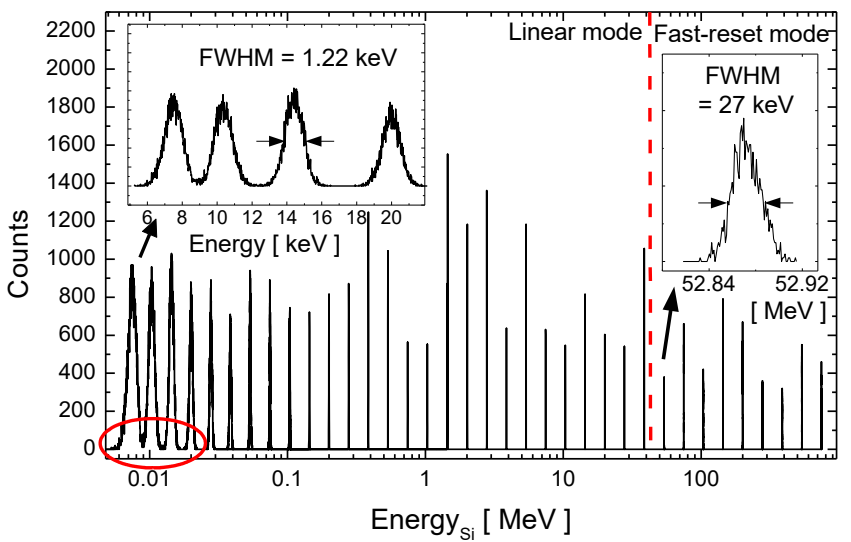

Fig. 10. Pulser lines from the back channel of the ASIC pre-amplifier as obtained in the linear mode (left) and the fast-reset mode (right). Note that the energy axis is in logarithmic scale. In the box in the upper-left corner the first four peaks are shown. The FWHM of the peak at $14.5 \mathrm{keV}$ is equal to $1.22 \mathrm{keV}$ which is adequate for the experimental requirements of the GALTRACE detector. The total input-referred capacitance is $\sim 5 \mathrm{pF}$.

For energies above the limit of $40 \mathrm{MeV}$ the pre-amplifier saturates and its output waveform can no longer be used for spectroscopic measurements. However, exploiting the properties of the fast-reset mechanism, a solution was worked out yielding reliable spectroscopic measurements even in this regime. The fast-reset device of the back channel, in fact, provides a square pulse, the time width of which is proportional to the charge removed from the input node. Such square pulses were acquired with a digital oscilloscope and their widths evaluated on a pulse-by-pulse basis using a simple Time-over-Threshold analysis. Every peak in the "fastreset" region is obtained by histogramming the widths of a thousand such waveforms. Each spectral line is spread over about 100 bins around the centroid. The data collected with this new procedure were then calibrated and added to the low-energy part of the spectrum, so as to obtain the joint spectrum of Fig. 10 which yields an exceptionally wide linear range of $103 \mathrm{~dB}$. The FWHM of the fast-reset peaks is in the order of $0.1 \%$ of the total energy or better. The residuals, calculated as deviations of the centroids from the best-fitting nine peaks equally spaced on an exponential scale, are smaller than $\pm 0.25 \%$.

\section{CONCLUSIONS}

A five-channel ASIC charge-sensitive pre-amplifier was designed, realized and tested (See Fig. 11 and table II). The power consumption is around $11 \mathrm{~mW}$ per channel and the noise performance meets the GAL-TRACE specifications. An equivalent noise charge in the order of 130 electrons r.m.s. ensures a resolution of $1.1 \mathrm{keV}$ in silicon for signals under the saturation threshold of the pre-amplifier. A signal risetime (10\%-90\%) of approximately $10 \mathrm{~ns}$ enables to perform pulse-shape analysis on waveforms to discriminate light ions impinging on silicon detectors. Given the valuable noise performance, this pre-amplifier is suitable also for gamma spectroscopy with germanium detectors.

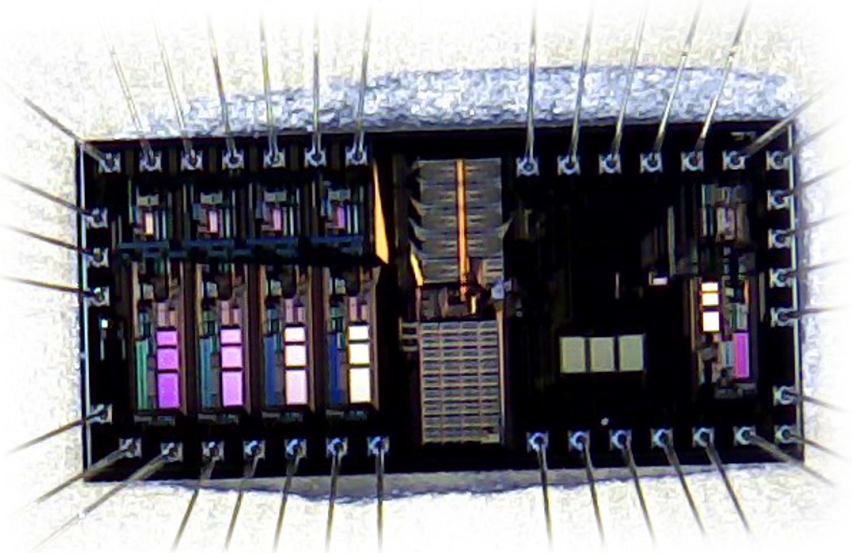

Fig. 11. Photograph of the $3.3 \mathrm{~mm} \times 1.5 \mathrm{~mm}$ chip wire-bonded in the cavity of a PLCC44 carrier. This image was taken with a magnifying camera connected to a PC through USB communication. The four blocks on the left are the anodic channels, the block on the right is the cathodic one and the structure in the middle is the $\mathrm{I}^{2} \mathrm{C}$ receiver

Thanks to the fast-reset mechanism, this pre-amplifier is capable of reducing the saturation dead time down to some microseconds (within realistic energy depositions in the detector). Beside this valuable feature, the fast-reset can be used to perform gamma-grade spectroscopy (with resolution of $0.2 \%$ FWHM or better) on an energy range that extends by more than one order of magnitude over the natural pre-amplifier dynamic range. When the pre-amplifier is saturated, the energy information is retrieved with a time-over-threshold technique that involves the measurement of the reset process duration. Thanks to this innovative technique the proposed preamplifer has a dynamic energy range of $103 \mathrm{~dB}$.

Although already known in literature, our implementation of the fast-reset concept shows better performance than previous works. Most of them implement a pulsed-reset technique only for dead-time reduction [22], [23], without the additional feature of the dynamic-range extension. If the baseline fluctuations are not negligible, the measurement of the reset time alone is not sufficient to retrieve a good energy information: digital [9] or analog [21] techniques must be used.

The chip is powered with a dual $\pm 2.5 \mathrm{~V}$ power rail. The overall area occupation is $5 \mathrm{~mm}^{2}$.

\section{ACKNOWLEDGMENT}

The authors would like to thank all the members of the GRIT (Gaspard-TRACE) European collaboration [24] [25].

\section{REFERENCES}

[1] M. Gelain, D. Mengoni, S. Lunardi, J. Dueas, and M. Assie, "Characterization of a highly-segmented silicon prototype for the trace array," vol. 66, 2014.

[2] J. Simpson, "The agata spectrometer: Next generation gamma-ray spectroscopy," vol. 606, no. 1, 2015.

[3] C. Ur, "Perspectives for the gamma-ray spectroscopy at LNL: The GALILEO project," vol. 366, no. 1, 2012.

[4] A. Andrighetto, M. Manzolaro, S. Corradetti, D. Scarpa, A. Monetti, M. Rossignoli, M. Ballan, F. Borgna, F. D’Agostini, F. Gramegna, G. Prete, G. Meneghetti, M. Ferrari, and A. Zenoni, "Spes: An intense source of neutron-rich radioactive beams at legnaro," vol. 966, no. 1, 2018. 
TABLE II

SUMMARY OF THE ASIC PARAMETERS.

\begin{tabular}{|c|c|}
\hline Process & $\begin{array}{c}\text { AMS } 0.35 \mu \mathrm{m} \\
\text { 3.3/5V Mixed Signal }\end{array}$ \\
\hline Die size & $3.3 \times 1.5 \mathrm{~mm}^{2}$ \\
\hline Power supply & $\pm 2.5 \mathrm{~V}$ \\
\hline Power consumption & $11 \mathrm{~mW} /$ channel \\
\hline Input stage structure & Differential \\
\hline $\begin{array}{l}\text { Number of channels } \\
\text { for anodic signals }\end{array}$ & 4 \\
\hline $\begin{array}{l}\text { Number of channels } \\
\text { for cathodic signals }\end{array}$ & 1 \\
\hline $\begin{array}{l}\text { ENC } \\
\text { (anodic channels) }\end{array}$ & $\begin{array}{c}130 \mathrm{e}^{-} \text {rms } \\
\text { (4 pF detector cap) }\end{array}$ \\
\hline $\begin{array}{l}\text { ENC } \\
\text { (cathodic cannels) }\end{array}$ & $\begin{array}{c}143 \mathrm{e}^{-} \text {rms } \\
\text { (4 pF detector cap) }\end{array}$ \\
\hline $\begin{array}{l}\text { Energy resolution } \\
\text { (anodic channels) }\end{array}$ & $\begin{array}{c}1.11 \mathrm{keV} \text { FWHM } \\
(4 \mathrm{pF})\end{array}$ \\
\hline $\begin{array}{l}\text { Energy resolution } \\
\text { (cathodic channels) }\end{array}$ & $\begin{array}{c}1.2 \mathrm{keV} \text { FWHM } \\
(4 \mathrm{pF})\end{array}$ \\
\hline Selectable gains & $0.2 / 0.5 / 0.7 / 1.0 \mathrm{mV} / \mathrm{fC}$ \\
\hline Linear dynamic range & $8 / 20 / 28 / 40 \mathrm{MeV}$ \\
\hline Fast-reset dynamic range & $>=700 \mathrm{MeV}$ \\
\hline rise-time $(10 \%-90 \%)$ & $9 \mathrm{~ns}$ minimum $(4 \mathrm{pF})$ \\
\hline
\end{tabular}

[5] R. Catherall et al., "The isolde facility," Journal of Physics G: Nuclear and Particle Physics, vol. 44, no. 9, 2017.

[6] M. Assi, A. Matta, B. Le Crom, M. Chabot, D. Mengoni, D. Beaumel, Y. Blumenfeld, J.-J. Dormard, B. Genolini, F. Hammache, J. Guillot, A. Jallat, E. Rauly, N. de Srville, and D. Suzuki, "New methods to identify low energy 3 he with silicon-based detectors," Nuclear Instruments and Methods in Physics Research, Section A: Accelerators, Spectrometers, Detectors and Associated Equipment, vol. 908, pp. 250255, 2018.

[7] D. Mengoni, J. Dueñas, M. Assi, C. Boiano, P. John, R. Aliaga, D. Beaumel, S. Capra, A. Gadea, V. Gonzles, A. Gottardo, L. Grassi, V. Herrero-Bosch, T. Houdy, I. Martel, V. Parkar, R. Perez-Vidal, A. Pullia, E. Sanchis, A. Triossi, and J. V. Dobn, "Digital pulse-shape analysis with a trace early silicon prototype," Nuclear Instruments and Methods in Physics Research Section A: Accelerators, Spectrometers, Detectors and Associated Equipment, vol. 764, pp. 241 - 246, 2014.

[8] S. Capra, D. Mengoni, R. Aliaga, A. Gadea, V. Gonzalez, and A. Pullia, "Design of an integrated low-noise, low-power charge sensitive preamplifier for gamma and particle spectroscopy with solid state detectors," 2014 IEEE Nuclear Science Symposium and Medical Imaging Conference, NSS/MIC 2014, 2014.

[9] S. Capra, D. Mengoni, J. Dueas, P. John, A. Gadea, R. Aliaga, J. Dormard, M. Assie, and A. Pullia, "Performance of the new integrated frontend electronics of the trace array commissioned with an early silicon detector prototype," Nuclear Instruments and Methods in Physics Research Section A: Accelerators, Spectrometers, Detectors and Associated Equipment, 2019.

[10] Europractice ams technologies. [Online]. Available: http://www. europractice-ic.com/technologies_ams2.php

[11] S. Capra, "Impedance and noise closed-form model of large-area integrated resistors with high stray capacitance to be used as feedback discharge devices in charge-sensitive preamplifiers for nuclear spectroscopy," IEEE Transactions on Nuclear Science, vol. 67, no. 4, pp. 722-731, 2020.

[12] A. Pullia, F. Zocca, G. Pascovici, and D. Bazzacco, "Extending the dynamic range of nuclear pulse spectrometers," Review of Scientific Instruments, vol. 79, no. 3, 2008.

[13] A. Pullia, C. Boiano, R. Bassini, and G. Pascovici, "Active reset of digitized preamplifiers for ionizing-radiation sensors," IEEE Transactions on Nuclear Science, vol. 51, no. 3 III, pp. 831-835, 2004.

[14] F. Zocca, A. Pullia, D. Bazzacco, and G. Pascovici, "A time-overthreshold technique for wide dynamic range gamma-ray spectroscopy with the agata detector," Nuclear Science, IEEE Transactions on, vol. 56, no. 4, pp. 2384-2391, Aug 2009.

[15] D. Przyborowski, J. Kaplon, and P. Rymaszewski, "Design and performance of the BCM1F front end asic for the beam condition monitoring system at the CMS experiment," IEEE Transactions on Nuclear Science, vol. 63, no. 4, pp. 2300-2308, 2016.

[16] T. Petrovi, M. Vencelj, M. Lipoglavek, R. Novak, and D. Savran, "Efficient reduction of piled-up events in gamma-ray spectrometry at high count rates," IEEE Transactions on Nuclear Science, vol. 61, no. 1 , pp. 584-589, 2014.

[17] A. Pullia and F. Zocca, "Fast low-impedance output stage for CMOS charge preamplifiers able to work at cryogenic temperatures," IEEE Nuclear Science Symposium Conference Record, pp. 353-356, 2009.

[18] A. Pullia, F. Zocca, and S. Capra, "Note: A 102 db dynamic-range charge-sampling readout for ionizing particle/radiation detectors based on an application-specific integrated circuit (asic)," Review of Scientific Instruments, vol. 89, no. 2, 2018.

[19] A. Pullia, F. Zocca, and M. Citterio, "VLSI cryogenic front-end for HPGe detectors based on a silicon-germanium technology," IEEE Nuclear Science Symposium Conference Record, pp. 1340-1342, 2010.

[20] F. Zocca, A. Pullia, S. Riboldi, A. D'Andragora, and C. Cattadori, "Setup of cryogenic front-end electronic systems for germanium detectors readout," IEEE Nuclear Science Symposium Conference Record, pp. 368$372,2009$.

[21] S. Capra and A. Pullia, "Experimental performance of a highlyinnovative low-noise charge-sensitive preamplifier with integrated rangebooster," Journal of Instrumentation, accepted for publication, 2018.

[22] M. Pftzner et al., "Charged-particle spectroscopy in the microsecond range following projectile fragmentation," Nuclear Instruments and Methods in Physics Research, Section A: Accelerators, Spectrometers, Detectors and Associated Equipment, vol. 493, no. 3, pp. 155-164, 2002.

[23] Z. Deng, L. He, F. Liu, Y. Liu, Y. Li, and Q. Yue, "An ultra-low noise cryogenic CMOS charge sensitive preamplifier for large volume pointcontact HPGe detectors," Journal of Instrumentation, vol. 13, no. 8, 2018.

[24] D. Beaumel, "The GASPARD project," Nucl. Instrum. Meth., vol. B317, pp. 661-663, 2013.

[25] "The TRACE array." [Online]. Available: https://web.infn.it/spes/index. php/research-on-nuclear-physics/150-trace 